\title{
Tract-Based Spatial Statistics of the Corpus Callosum using Different Tensor-Derived Indices
}

\author{
Thomas van Bruggen ${ }^{1}$, Bram Stieltjes ${ }^{2}$, Hans-Peter Meinzer ${ }^{1}$, \\ Klaus H. Fritzsche ${ }^{1,2}$ \\ ${ }^{1}$ German Cancer Research Center, Div. of Medical and Biological Informatics \\ ${ }^{2}$ German Cancer Research Center, Div. of Radiology, Sect. Quantitative \\ Imaging-based Disease Characterization \\ t. vanbruggen@dkfz-heidelberg.de
}

\begin{abstract}
Prior work has shown that white matter fiber integrity decreases in Alzheimer's disease (AD) and mild cognitive impairment (MCI). This integrity can be quantified using diffusion tensor imaging techniques, which describe the anisotropic water movement in the brain. It is important to identify features that can predict the chance of conversion from MCI to AD within a certain time frame. In this study we applied tract-based spatial statistics (TBSS) in order to perform this task, overcoming limitations that are commonly associated with ROI-based approaches and voxel-based morphometry (VBM). Diffusion weighted images were taken from 15 healthy controls, $15 \mathrm{AD}$ patients and $17 \mathrm{MCI}$ patients. 8 MCI patients remained stable during 3 year follow-up investigations ("non-converters", MCI-nc) and 9 converted to AD ("converters", MCI-c). Significant differences between the MCI-nc and MCI-c groups were found in a large part of the corpus callosum using fractional anisotropy (FA) and radial diffusivity. In comparison, the MCI-c group did not differ significantly from the AD group and the MCI-nc group exhibited similar measurements as the control group. These results demonstrate that, although MCI-c and MCI-nc patients were clinically similar at time of inclusion, the MCI-c group already exhibited pathologic features associated with $\mathrm{AD}$. This finding could lead to more powerful techniques in the early identification of $\mathrm{AD}$ and thus support an earlier and more successful treatment.
\end{abstract}

\section{Introduction}

Diffusion tensor imaging (DTI) is an advanced MRI technique that provides information about the fiber architecture of the brain by measuring the movement of water protons. In white matter, the diffusion perpendicular to the fibers is lower than parallel to the fibers. The degree of anisotropy can be related to the tract integrity. Assuming that the displacement distribution is Gaussian, diffusion can be described by a tensor, a $3 \times 3$ matrix that describes the diffusion in $3 D$ space. The direction of maximum diffusion, the principal direction, corresponds to the first eigenvector, which is the direction parallel to the fiber 
direction. Together with the eigenvalues the eigenvectors describe the properties of the tensor. The eigenvalues are ordered as $\lambda_{1} \geq \lambda_{2} \geq \lambda_{3}$ [1].

Recent studies demonstrated a decrease of white matter integrity in MCI and AD patients compared to healthy controls $[2,3])$. However, less than half of all MCI patients convert to $\mathrm{AD}$ [4], making it important to identify markers with a predictive value of the chance that an MCI patient will develop $\mathrm{AD}$ within a certain time frame. Such markers would allow early and more successful medical treatment and might increase our understanding of the early pathology in AD. Most studies base their analysis on the evaluation of regions of interest (ROIs) or voxel-based morphometry (VBM) [5]. The disadvantage of ROI-based methods is the difficulty to objectively place the ROI, whereas VBM is impaired by spatial alignment errors and partial volume effects. In this study we applied tractbased spatial statistics (TBSS), which is a new method that aims at overcoming these obstacles by reducing the data to a white matter skeleton. Here only the highest FA values per tract are identified for statistical analysis in order to obtain pure white matter measurements and to omit areas that contain partial volumes while at the same time correcting for small registration errors [6]. Other studies already found lower white matter integrity in patients with $\mathrm{AD}$ and MCI using this technique $[3,2,7]$. In this study we used TBSS to look at the differences between MCI patients that converted to $\mathrm{AD}$ within a time frame of three years and MCI patients that remained stable in this period.

\section{Materials and Methods}

47 subjects were recruited, including 15 controls, $18 \mathrm{MCI}$ patients and $15 \mathrm{AD}$ patients with mean ages of $66( \pm 7)$ years, $70( \pm 5)$ years, and $72( \pm 7)$ years, respectively. The clinical evaluation of all subjects included ascertainment of personal and family history as well as physical, neurological, and neuropsychological examination. Those with a history of ischemic heart disease, cancer, and cerebrovascular risk factors were excluded. MCI was defined by Levy's criteria of Aging-associated cognitive decline. Mild to moderate Alzheimer's disease was defined by the NINCDS-ADRDA-criteria. A clinical follow-up of the patients was done within three years after inclusion. From the MCI patients 8 remained stable and 9 converted to $\mathrm{AD}$ in this period. At the time of inclusion, the MMS of the AD group was 19.2, that of the healthy control group was 29.3, and that of the whole MCI group was 26.4. The MCI-nc group and the MCI-c group showed a comparable MMS of 26.8 and 26.2. After three years, the MME of the MCI-nc was 26.9. The MME of the MCI-c group, however, decreased significantly to 23.3 (Mann-Whitney: $\mathrm{p}=0.006$ ). Diffusion weighed imaging was performed on a $1.5 \mathrm{~T}$ whole body clinical scanner and a quadrature head coil (Magnetom Symphony, Siemens Medical Solutions, Erlangen, Germany) with a gradient strength of $40 \mathrm{mT} / \mathrm{m}$. A single shot echoplanar imaging technique with a twice refocused spin echo diffusion preparation was employed using the following parameters: TR/Echo Time (TE) 4700/78 ms, field of view $240 \mathrm{~mm}$, data matrix of $96 \times 96$ yielding an in plane resolution of $2.5 \mathrm{~mm}, 50$ axial slices with 
a thickness of $2.5 \mathrm{~mm}$ and no gap, $\mathrm{N}=12$ gradient directions and two b-values $(0$ and $1000 \mathrm{~s} / \mathrm{mm} 2)$. In order to increase the stability, 5 subsequent Diffusion Tensor Imaging (DTI) datasets were acquired, spatially matched and averaged. Diffusion tensors were estimated using a linear least squares fit and FA values were calculated for all subjects, using

$$
F A=\sqrt{\frac{3}{2}} \sqrt{\frac{\left(\lambda_{1}-\bar{\lambda}\right)^{2}+\left(\lambda_{2}-\bar{\lambda}\right)^{2}+\left(\lambda_{3}-\bar{\lambda}\right)^{2}}{\lambda_{1}^{2}+\lambda_{2}^{2}+\lambda_{3}^{2}}}
$$

where $\bar{\lambda}$ is the mean of the eigenvectors. Also the axial $\lambda_{1}$ and radial $\frac{\lambda_{2}+\lambda_{3}}{2}$ diffusivity were calculated. [1]. The brain masks were extracted from the nondiffusion-weighted images using BET (Brain Extraction Tool), which is available in the FSL package [8] and used to mask the FA-images. This way all voxels that did not belong to the cranial volume were masked out. The masked FA-images were registered linearly followed by non-linear registration to the FMRIB58 template using the FLIRT and FNIRT tools (the template and both registration tools are also available in the FSL package). In order to obtain a binary mask of voxels that are positioned in the tracts centers of the averaged FA-image, the mean FA-image was thinned using the TBSS skeletonization tool and thresholded with an FA of 0.2 [6]. The resulting images of this skeletonization process are shown in Figure 1. Each subject's spatially aligned dataset was then projected onto the binary mask in order to obtain individual measurements that correspond to skeleton positions. The images with the axial and radial diffusivity measurements were transformed with the transformation parameters that were acquired while registering the FA images and measurements for statistical analysis were taken from the same image locations as the FA values. The resulting individual measurements were now used to perform voxel-wise group statistics on the corpus callosum. This structure was chosen since it is a very distinctive structure which is robustly quantifiable and plays an important role in many neurodegenerative diseases. Figure 1 shows a segmentation of this structure. It was segmented manually from the binary skeleton using MITK (www.mitk.org). FA, axial diffusivity and radial diffusivity along the corpus callosum were read out in the anterior-posterior direction and group statistics were performed by means of t-tests using a significance level of $\alpha=0.05$.

\section{Results}

Figure 2a-c show the mean FA values, the axial diffusivities, and radial diffusivities on the corpus callosum in the anterior-posterior direction. The results of the t-tests are summarized in diagrams under each respective graph as well (Fig. 2d-f). The results are reported for the group comparisons controls/MCI-nc, MCI-nc/MCI-c and the MCI-c/AD, since these are adjacent pairs when ordering the groups by disease severity. When looking at the FA and radial diffusivity, the healthy controls and the MCI-nc group have similar values and the MCI-c and the AD group also. for the axial diffusivity this distinction is less clear. The 
MCI-nc/MCI-c group comparison shows significant differences in FA and radial diffusivity on many positions along the corpus callosum and only very few positions with significant differences for both other comparisons. However, for the axial diffusivity there are much less positions showing a significant difference on the MCI-nc/MCI-c group comparison and more on the controls/MCI-nc group comparison.

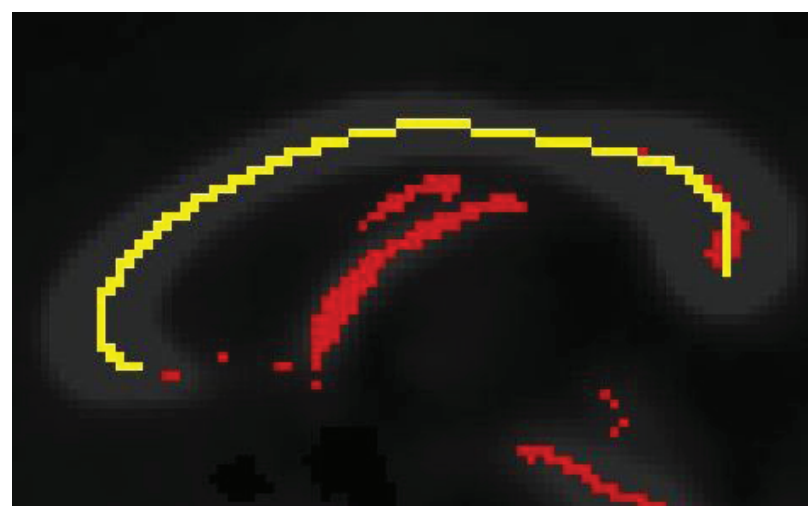

Fig. 1. A mean FA image was obtained by spatially normalizing and averaging the FA images of all subjects. Using TBSS, a FA skeleton was extracted from this mean FA image and a binary skeleton was obtained by thresholding the mean FA skeleton. The corpus callosum was segmented manually from the binary skeleton. Here a sagittal slice is shown of the mean FA image (white) with the binary skeleton overlaid in red and the segmentation of the corpus callosum overlaid in yellow.

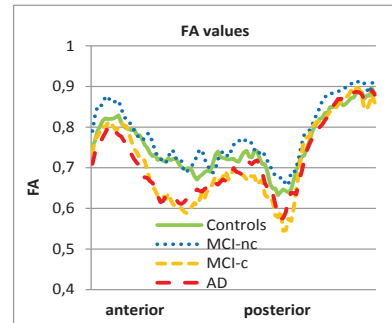

(a)

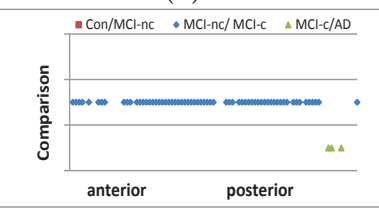

(d)

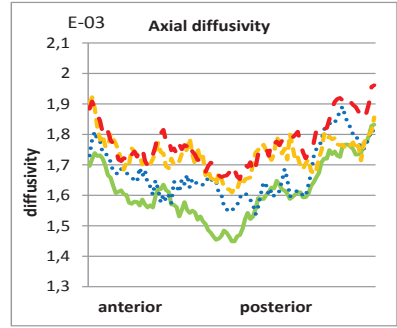

(b)

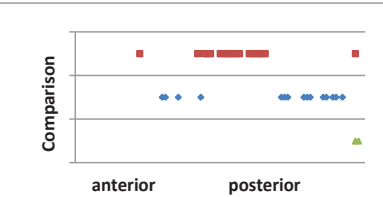

(e)

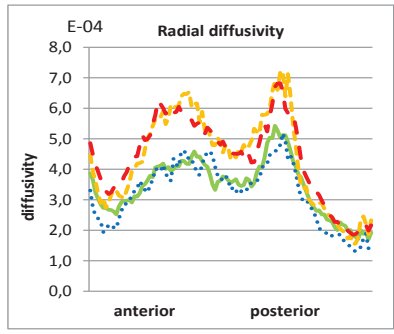

(c)

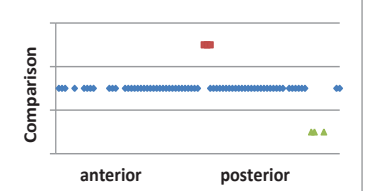

(f)

Fig. 2. The FA values (a), the axial diffusivities (b) and the radial diffusivities (c) in the anterior-posterior direction in the corpus callosum. Figures (d) to (f) indicate positions with a significant difference $(p<0.05)$, between the groups under comparison. 


\section{Discussion}

In this work we performed tract-specific analysis of diffusion on the corpus callosum, which is a structure that plays an important role in neurodegenerative diseases. In addition to other studies that focus on the differences between AD patients and healthy controls, we retrospectively looked at MCI data, trying to find early markers for the development of AD. Significant differences between MCI-nc and MCI-c were found at nearly all positions from splenium to genu of the corpus callosum when using FA and radial diffusivity as a measurement for tract integrity, indicating that these provide early pathologic hallmarks of $\mathrm{AD}$ that are clearly manifested in the diffusion images of MCI-c patients and are absent in those of MCI-nc patients. This is an important result since current clinical tests fail to separate the converting and non-converting MCI subjects at the time of inclusion. Furthermore, hardly any differences in FA or radial diffusivity appeared when comparing the MCI-c group with the AD group, indicating that these groups have similar fiber integrity. The same counts when comparing the healthy control group with the MCI-nc group. When using axial diffusivity as a measure the number of significant positions for the MCI-nc/MCI-c comparison was lower, whereas the number of significant positions for the controls/MCI-nc comparison increased, suggesting that this property is affected even earlier in the course of the disease progression. The results presented in this paper demonstrate the possibility of defining early predictors for Alzheimer's disease. We showed that radial diffusivity and fractional anisotropy carry information that can help to predict the chances of conversion from MCI to AD, which is very important for early treatment and therapy planning.

\section{References}

1. Hagmann P, Jonasson L, Meader P, et al. Understanding diffusion MR imaging techniques: From scalar diffusion-weighted imaging to diffusion tensor imaging and beyond. Radiographics. 2006;26:205-223.

2. Damoiseaux JS, Smith SM, Witter MP, et al. White matter tract integrity in aging and Alzheimer's disease. Hum Brain Mapp. 2009;30(4):1051-59.

3. Salat DH, Tuch DS, van der Kouwe AJW, et al. White matter pathology isolates the hippocampal formation in alzheimer's disease. Hum Brain Mapp. 2010;31(2):244-56.

4. Mitchell AJ, Shiri-Feshki M. Rate of progression of mild cognitive impairment to dementia. Acta Psychiatr Scand. 2009;119(4):252-65.

5. Ashburner J, Friston KJ. Voxel-based morphometry: the methods. Neuroimage. 2000;11:805-51.

6. Smith SM, Jenkinson M, Rueckert D, et al. Tract-based spatial statistics: voxelwise analysis of multi-subject diffusion data. Neuroimage. 2006;31(4):1487-505.

7. Liu Y, Spulber G, Lehtimäki KK, et al. Diffusion tensor imaging and tract-based spatial statistics in alzheimer's disease and mild cognitive impairment. Neurobiol Aging. 2009.

8. Smith SM, Jenkinson M, Woolrich MW, et al. Advances in functional and structural MR image analysis and implementation as FSL. Neuroimage. 2004;23:208-19. 